\title{
Connectedness concept in intuitionistic fuzzy topological spaces
}

\author{
Md. Aman Mahbub ${ }^{1, *}$, Md. Sahadat Hossain ${ }^{2}$ \\ and M. Altab Hossain ${ }^{3}$ \\ ${ }^{1}$ Department of Mathematics, Comilla University, Comilla-3506, Bangladesh \\ e-mail: rinko.math@gmail.com \\ ${ }^{2}$ Department of Mathematics, University of Rajshahi, Rajshahi, Bangladesh \\ e-mail: sahadateru.ac.bd \\ ${ }^{3}$ Department of Mathematics, University of Rajshahi, Rajshahi, Bangladesh \\ e-mail: al_math_bderu.ac.bd \\ * Corresponding author
}

Received: 28 December 2020

Accepted: 7 March 2021

\begin{abstract}
The purpose of this paper is to establish the connectedness in intuitionistic fuzzy topological space. In this paper we give six notions of separatedness, connectedness and total connectedness and one notion of $T_{1}$-space in intuitionistic fuzzy topological space. Also, we find a relation between classical topology and intuitionistic fuzzy topology. Further, we show that connectedness in intuitionistic fuzzy topological spaces are productive and we demonstrate some of its features.
\end{abstract}

Keywords: Fuzzy set, Intuitionistic fuzzy set, Intuitionistic topological space, Intuitionistic fuzzy topological space, Intuitionistic fuzzy connectedness, Intuitionistic fuzzy $T_{1}$-space.

2020 Mathematics Subject Classification: 03E72.

\section{Introduction}

The basic concept of a fuzzy set was given by Zadeh [40] in 1965, after then fuzzy topology by Chang [13] in 1968. The generalized concept of intuitionistic fuzzy set was introduced by Atanassov [9] which take into account both the degrees of membership and non-membership subject to the condition that their sum does not exceed 1. Coker [11, 12, 14|-17] and his colleagues introduced intuitionistic fuzzy topological spaces and connectedness in intuitionistic 
fuzzy topological spaces was introduced by Ozcag and Coker [29]. Islam et al. [23, 24], S. Das [18], Lee et al. [25, 26], Minana et al. [28], M. Barile [10], R. Srivastava et al. [33, 34], Tiwari et al. [38], Estiaq Ahmed et al. [1-5], L. Ying-Ming et al. [39], Talukder et al. [35], Fang et al. [19], Hasan et al. [20], Tamilmani [37], R. Islam et al. [22], M. K. Ahmad et al. [6], A. M. Ali et al. [7, 8], Ramadan et al. [30], Immaculate et al. [21] and N. X. Tan et al. [36] subsequently developed the study of intuitionistic fuzzy topological spaces by using intuitionistic fuzzy sets. In this paper, we define six new notions of separatedness, connectedness and total connectedness and one notion of $T_{1}$-space in intuitionistic fuzzy topological space and some of their features.

\section{Notation and preliminaries}

Throughout this paper, $X$ is a non-empty set, $T$ is a topology, $t$ is a fuzzy topology, $\mathcal{T}$ is an intuitionistic topology and $\tau$ is an intuitionistic fuzzy topology. $\lambda$ and $\mu$ are fuzzy sets, $A=\left(\mu_{A}, v_{A}\right)$ is an intuitionistic fuzzy set. Particularly, by $\underline{0}$ and $\underline{1}$ we denote constant fuzzy sets taking values 0 and 1 , respectively.

Definition 2.1 [13]. Let $X$ be a non-empty set. A family $t$ of fuzzy sets in $X$ is called a fuzzy topology on $X$ if the following conditions hold.

(1) $\underline{0}, \underline{1} \in t$,

(2) $\lambda \cap \mu \in t$ for all $\lambda, \mu \in t$,

(3) $\cup \lambda_{j} \in t$ for any arbitrary family $\left\{\lambda_{j} \in t, j \in J\right\}$.

Definition 2.2 [14]. Suppose that $X$ is a non-empty set. An intuitionistic set $A$ on $X$ is an object having the form $A=\left(X, A_{1}, A_{2}\right)$, where $A_{1}$ and $A_{2}$ are subsets of $X$ satisfying $A_{1} \cap A_{2}=\phi$. The set $A_{1}$ is called the set of members of $A$, while $A_{2}$ is called the set of non-members of $A$. In this paper, we use the simpler notation $A=\left(A_{1}, A_{2}\right)$ instead of $A=\left(X, A_{1}, A_{2}\right)$ for an intuitionistic set.

Definition 2.3 [9]. Let $X$ be a non-empty set. An intuitionistic fuzzy set $A$ (IFS, in short) in $X$ is an object having the form $A=\left\{\left(x, \mu_{A}(x), v_{A}(x)\right): x \in X\right\}$, where $\mu_{A}$ and $v_{A}$ are fuzzy sets in $X$ denoting the degree of membership and the degree of non-membership, respectively, subject to the condition $\mu_{A}(x)+v_{A}(x) \leq 1$.

Throughout this paper, we use the simpler notation $A=\left(\mu_{A}, v_{A}\right)$ instead of $A=$ $\left\{\left(x, \mu_{A}(x), v_{A}(x)\right): x \in X\right\}$ for IFSs.

Definition 2.4 [9]. Let $X$ be a non-empty set and IFSs $A, B$ in $X$ be given by $A=\left(\mu_{A}, v_{A}\right)$ and $B=\left(\mu_{B}, v_{B}\right)$ respectively, then

(a) $A \subseteq B$ if $\mu_{A}(x) \leq \mu_{B}(x)$ and $v_{A}(x) \geq v_{B}(x)$ for all $x \in X$,

(b) $A=B$ if $A \subseteq B$ and $B \subseteq A$,

(c) $\bar{A}=\left(v_{A}, \mu_{A}\right)$,

(d) $A \cap B=\left(\mu_{A} \cap \mu_{B}, v_{A} \cup v_{B}\right)$,

(e) $A \cup B=\left(\mu_{A} \cup \mu_{B}, v_{A} \cap v_{B}\right)$. 
Definition 2.5 [14]. Let $\left\{A_{j}=\left(\mu_{A_{j}}, v_{A_{j}}\right), j \in J\right\}$ be an arbitrary family of IFSs in $X$. Then

(a) $\cap A_{j}=\left(\cap \mu_{A_{j}}, \cup v_{A_{j}}\right)$,

(b) $\cup A_{j}=\left(\cup \mu_{A_{j}}, \cap v_{A_{j}}\right)$,

(c) $0_{\sim}=(\underline{0}, \underline{1}), 1_{\sim}=(\underline{1}, \underline{0})$.

Definition 2.6 [14]. An intuitionistic fuzzy topology (IFT, in short) on a non-empty set $X$ is a family $\tau$ of IFSs in $X$ satisfying the following axioms:

(1) $0_{\sim}, 1_{\sim} \in \tau$,

(2) $A \cap B \in \tau$, for all $A, B \in \tau$,

(3) $\cup A_{j} \in \tau$ for any arbitrary family $\left\{A_{j} \in \tau, j \in J\right\}$.

The pair $(X, \tau)$ is called an intuitionistic fuzzy topological space (IFTS, in short), members of $\tau$ are called intuitionistic fuzzy open sets (IFOS, in short).

Definition 2.7 [32]. Let $A=\left(\mu_{A}, v_{A}\right)$ be an IFS in $X$ and $U$ be a non-empty subset of $X$. The restriction of $A$ to $U$ is an IFS in $U$, denoted by $A \mid U$ and defined by $A \mid U=\left(\mu_{A}\left|U, v_{A}\right| U\right)$.

Definition 2.8 [23]. Let $(X, \tau)$ be an intuitionistic fuzzy topological space and $U$ be a non-empty subset of $X$, then $\tau_{U}=\{A \mid U: A \in \tau\}$ is an intuitionistic fuzzy topology on $U$ and $\left(U, \tau_{U}\right)$ is called subspace of $(X, \tau)$.

Definition 2.9 [32]. Let $\alpha, \beta \in(0,1)$ and $\alpha+\beta \leq 1$. An intuitionistic fuzzy point (IFP for short) $p_{(\alpha, \beta)}^{x}$ of $X$ defined by $p_{(\alpha, \beta)}^{x}=\left\langle x, \mu_{p}, v_{p}\right\rangle$, for $y \in X$

$$
\mu_{p}(y)=\left\{\begin{array}{lll}
\alpha & \text { if } & y=x \\
0 & \text { if } & y \neq x
\end{array} \text { and } v_{p}(y)=\left\{\begin{array}{lll}
\beta & \text { if } & y=x \\
1 & \text { if } & y \neq x
\end{array}\right.\right.
$$

In this case, $x$ is called the support of $p_{(\alpha, \beta)}^{x}$. An IFP $p_{(\alpha, \beta)}^{x}$ is said to belong to an IFS $A=\left\langle x, \mu_{A}, v_{A}\right\rangle$ of $X$, denoted by $p_{(\alpha, \beta)}^{x} \in A$, if $\alpha \leq \mu_{A}(x)$ and $\beta \geq v_{A}(x)$.

Proposition 2.1 [32]. An IFS A in $X$ is the union of all IFP belonging to A.

Definition 2.10 [11]. Let $A=\left(x, \mu_{A}, v_{A}\right)$ and $B=\left(y, \mu_{B}, v_{B}\right)$ be IFSs in $X$ and $Y$ respectively. Then the product of IFSs $A$ and $B$ denoted by $A \times B$ is defined by $A \times B=$ $\left.\left\{(x, y), \mu_{A} \stackrel{\times}{.} \mu_{B}, v_{A} \times v_{B}\right)\right\}$ where $\left(\mu_{A} \stackrel{\times}{.} \mu_{B}\right)(x, y)=\min \left(\mu_{A}(x), \mu_{B}(y)\right)$ and $\left(v_{A} \cdot v_{B}\right)(x, y)=$ $\max \left(v_{A}(x), v_{B}(y)\right)$ for all $(x, y) \in X \times Y$. Obviously, $0 \leq\left(\mu_{A}{ }^{\times} \mu_{B}\right)+\left(v_{A} \times v_{B}\right) \leq 1$. This definition can be extended to an arbitrary family of IFSs.

Definition 2.11 [34]. Two disjoint non-empty intuitionistic fuzzy subsets $A=\left(\mu_{A}, v_{A}\right)$ and $B=\left(\mu_{B}, v_{B}\right)$ of an IFTS $(X, \tau)$ are said to be separated if there exist $U_{i} \in \tau(i=1,2)$ such that $U_{1} \supseteq A, U_{2} \supseteq B$ and $U_{1} \cap A=U_{2} \cap B=0 \sim$.

Definition 2.12 [34]. Let $(X, \tau)$ be an IFTS and $A$ be an IFS in $X$ which is strictly positive, i.e., $A(x) \gg 0_{\sim}$ (i.e., $\left.\mu_{A}(x)>0, v_{A}(x)<1, \forall x \in X\right)$. A pair $U_{1}, U_{2} \in \tau$ is called $\left(C_{1}\right)$-separation of $A$ if $U_{1} \neq A, U_{2} \neq A, U_{1} \cup U_{2}=A$ and $U_{1} \cap U_{2}=0_{\sim}$. 
Definition 2.13 [31]. A fuzzy topological space $X$ is said to be disconnected if $X=A \cup B$, where $A$ and $B$ are non-empty open fuzzy sets in $X$ such that $A \cap B=\emptyset$. Hence a fuzzy topological space $X$ cannot be represented as the union of two non-empty, disjoint open fuzzy sets on $X$.

Definition 2.14 [27]. Let $(X, \tau)$ be an intuitionistic fuzzy topological space. A family $\left\{\left(\mu_{G_{i}}, v_{G_{i}}\right): i \in J\right\}$ of IFOS in $X$ is called open cover of $X$ if $\cup \mu_{G_{i}}=1$ and $\cap v_{G_{i}}=0$. If every open cover of $X$ has a finite subcover, then $X$ is said to be intuitionistic fuzzy compact (IFcompact, in short).

Definition 2.15 [27]. A family $\left\{\left(\mu_{G_{i}}, v_{G_{i}}\right): i \in J\right\}$ of IFOS in $X$ is called $(\alpha, \beta)$-level open cover of $X$ if $\cup \mu_{G_{i}} \geq \alpha$ and $\cap v_{G_{i}} \leq \beta$ with $\alpha+\beta \leq 1$. If every $(\alpha, \beta)$-level open cover of $X$ has $a$ finite subcover, then $X$ is said to be $(\alpha, \beta)$-level IF-compact.

\section{Connectedness in intuitionistic fuzzy topological space}

In this section we define six new definitions of separatedness, connectedness and total connectedness and one notions of $T_{1}$-space in intuitionistic fuzzy topological space (IFTS, in short) and established several properties of these notions.

Definition 3.1. Two disjoint non-empty intuitionistic fuzzy subsets $A=\left(\mu_{A}, v_{A}\right)$ and $B=\left(\mu_{B}, v_{B}\right)$ of an IFTS $X$ are said to be separated if $A$ and $B$ neither contain a limit point of the other, i.e., $A$ and $B$ are separated iff $A \cap \bar{B}=(0,1)$ and $\bar{A} \cap B=(0.1)$.

Definition 3.2. Two IFS's $A=\left(\mu_{A}, v_{A}\right)$ and $B=\left(\mu_{B}, v_{B}\right)$ in $X$ are called $Q$-separated for an $\operatorname{IFTS}(X, \tau)$ if and only if there exist closed (open) IFS's $G=\left(\mu_{G}, v_{G}\right)$ and $H=\left(\mu_{H}, v_{H}\right)$ in $X$ such that $A \subseteq G, B \subseteq H$ and $A \cap B=(0,1)=G \cap H$.

Definition 3.3. An intuitionistic fuzzy subsets $A=\left(\mu_{A}, v_{A}\right)$ of an IFTS $X$ is disconnected if there exist open intuitionistic fuzzy subsets $G=\left(\mu_{G}, v_{G}\right)$ and $H=\left(\mu_{H}, v_{H}\right)$ of $X$ such that $(A \cap G) \cup(A \cap H)=(1,0)$ and $(A \cap G) \cap(A \cap H)=(0,1)$. In this case, $G \cup H$ is called a disconnection.

Definition 3.4. An IFTS $X$ is said to be disconnected if $A \cup B=(1,0)$ and $A \cap B=(0,1)$ where $A=\left(\mu_{A}, v_{A}\right)$ and $B=\left(\mu_{B}, v_{B}\right)$ are non-empty open intuitionistic fuzzy subsets of $X$.

Theorem 3.1. Union of two non-empty separated intuitionistic fuzzy subsets of an IFTS $X$ is disconnected.

Proof. Let $A=\left(\mu_{A}, v_{A}\right)$ and $B=\left(\mu_{B}, v_{B}\right)$ are two non-empty separated intuitionistic fuzzy subsets of an IFTS $X$, so $A \cap \bar{B}=(0,1)$ and $\bar{A} \cap B=(0.1)$. Let $G=\bar{B}^{C}$ and $H=\bar{A}^{C}$. Then $G$ and $H$ are open and $(A \cup B) \cap G=\left(1_{\mathrm{A}}, 0\right)$ and $(A \cup B) \cap H=\left(1_{\mathrm{B}}, 0\right)$ are non-empty disjoint IFSs whose union is $A \cup B$. Thus $G$ and $H$ form a disconnection of $A \cup B$. Hence $A \cup B$ is disconnected.

Theorem 3.2. Consider $\mathcal{M}=\left\{A_{i}\right\}$, where $A_{i}=\left(\mu_{A_{i}}, v_{A_{i}}\right)$ is a class of IF-connected subsets of an IFTS $X$ such that no two members of $\mathcal{M}$ are separated. Then $B=\cup_{i} A_{i}$ is IF-connected. 
Proof. Assume that $B$ is not IF-connected. Let $G=\left(\mu_{G}, v_{G}\right)$ and $H=\left(\mu_{H}, v_{H}\right)$ are two open IFS of $X$ such that $G \cup H$ is an IF-disconnection of $B$. Now each $A_{i} \in \mathcal{M}$ is IF-connected and so is contained in either $G$ or $H$ and disjoint from the other. Since any two members of $A_{i_{1}}, A_{i_{2}} \in \mathcal{M}$ are not separated and so $A_{i_{1}} \cup A_{i_{2}}$ is IF-connected, hence $A_{i_{1}} \cup A_{i_{2}}$ is contained in either $G$ or $H$ and disjoint from the other. Accordingly all the members of $\mathcal{M}$ and hence $B=\cup_{i} A_{i}$ must be contained in either $G$ or $H$ and disjoint from the other. But this contradicts the fact that $G \cup H$ is an IF-disconnection of $B$, hence $B$ is IF-connected.

Theorem 3.3. Let $G \cup H$ be a disconnection of an IFS $A=\left(\mu_{A}, v_{A}\right)$. Then $A \cap G$ and $A \cap H$ are separated IFSs.

Proof. Here $A \cap G$ and $A \cap H$ are disjoint, hence we need only to show that each IFS contains no limit point of the other. Let $p_{(m, n)}, m, n \in I$ be a limit point of $A \cap G$ and suppose $p_{(m, n)} \in A \cap$ $H$. Then $H$ is an open IFS containing $p_{(m, n)}$ and so $H$ contains a point of $A \cap G$ distinct from $p_{(m, n)}$, i.e., $(A \cap G) \cap H \neq(0,1)$. But $(A \cap G) \cap(A \cap H)=(0,1)=(A \cap G) \cap H$. Accordingly $p_{(m, n)} \notin A \cap H$. Similarly if $p_{(m, n)}$ be a limit point of $A \cap H$, then $p_{(m, n)} \notin A \cap G$. Thus $A \cap G$ and $A \cap H$ are separated IFSs.

Theorem 3.4. If an IFTS $(X, \tau)$ is IF-disconnected and $\tau^{*} \supseteq \tau$ then $\left(X, \tau^{*}\right)$ is also IFdisconnected.

Proof. Given IFTS $(X, \tau)$ is IF-disconnected. Let $A, B \in \tau$ where $A=\left(\mu_{A}, v_{A}\right)$ and $B=\left(\mu_{B}, v_{B}\right)$ are non-empty open intuitionistic fuzzy subsets of X, then $A \cup B=(1,0)$ and $A \cap B=(0,1)$. Since $\tau^{*} \supseteq \tau$ and $A, B \in \tau$ then obviously , $B \in \tau^{*}$, hence $A \cup B=(1,0)$ and $A \cap B=(0,1)$, which implies that $\left(X, \tau^{*}\right)$ is also IF-disconnected.

Definition 3.5. An IFTS $(X, \tau)$ is called

a) Intuitionistic fuzzy connected (IFC) (i) if $(X, \tau)$ has no proper clopen (clopen means closed-open) IFS.

b) IFC (ii) if there do not exist non-empty IFSs $A, B$ in $X$ which are separated and $A \cup B=(1,0)$.

c) IFC (iii) if there is no clopen IFS $A>>(0,1)$ which is $C 1$ separated.

d) IFC (iv) if there do not exist $A=\left(\mu_{A}, v_{A}\right), B=\left(\mu_{B}, v_{B}\right) \in \tau \backslash\{(0,1),(1.0)\}$ such that $A \cup B=(r, 0)$ with $0<r \leq 1$ and $A \cap B=(0,1)$.

e) IFC (v) iff for any $\alpha \in I_{0}$, there exist no non-empty proper subset $H \subseteq X$ such that $\alpha 1_{H}=\alpha\left(1_{H}, 1_{X-H}\right), \alpha 1_{X-H}=\alpha\left(1_{X-H}, 1_{H}\right) \in \tau$.

f) IFC (vi) iff there exist no non-zero $Q$-separated IFSs $A=\left(\mu_{A}, v_{A}\right)$ and $B=\left(\mu_{B}, v_{B}\right)$ in $X$ with $A \cup B=(1,0)$.

Theorem 3.5. The following statements are equivalent:

a) $\operatorname{IFTS}(X, \tau)$ is $\operatorname{IFC}(v i)$

b) There do not exist two non-zero disjoint closed IFSs $A=\left(\mu_{A}, v_{A}\right)$ and $B=\left(\mu_{B}, v_{B}\right)$, where $\max \left(\mu_{A}, \mu_{B}\right)=1$.

c) There do not exist two non-zero disjoint open IFSs $A=\left(\mu_{A}, v_{A}\right)$ and $B=\left(\mu_{B}, v_{B}\right)$, where $\max \left(\mu_{A}, \mu_{B}\right)=1$.

d) $\operatorname{IFTS}(X, \tau)$ is IFC (ii) 
Proof. (a) $\Rightarrow$ (b): Let there exist IFSs $A=\left(\mu_{A}, v_{A}\right), B=\left(\mu_{B}, v_{B}\right) \in \tau^{C}$ such that $A \neq B, A \cup B=$ $(1,0)$ and $A \cap B=(0,1)$ then clearly $A$ and $B$ are Q-separated. So that, $(X, \tau)$ is not IFC (vi), a contradiction to (a).

(b) $\Rightarrow$ (c): If $A, B \in \tau$ where $A=\left(\mu_{A}, v_{A}\right), B=\left(\mu_{B}, v_{B}\right), A \cup B=(1,0)$ and $A \cap B=(0,1)$ then $A$ and $B$ closed which contradicts (b).

(c) $\Rightarrow$ (d): If $(X, \tau)$ is not IFC (ii) then there exist $A, B \in I^{X}-\{(1,0)\}$ such that $A, B$ are separated and $A \cup B=(1,0)$. Now $\exists G, H \in \tau$ such that $A \subseteq G, B \subseteq H$ and $G \cap B=(1,0)=H \cap A$. But then $G$ and $H$ satisfying $G \cap H=(0,1)$ and $G \cup H=(1,0)$ which contradicting (c).

(d) $\Rightarrow$ (a): If there exist some IFS $A=\left(1_{A}, 1_{A} C\right) \in \tau \cap \tau^{C}-\{(0,1),(1,0)\}$, then $A=\left(1_{A}, 1_{A} C\right)$, $A^{C}=\left(1_{A} C, 1_{A}\right)$ are two non-zero separated sets with $\max \left(1_{A}, 1_{A^{C}}\right)=1$. This contradicts (d).

Theorem 3.6. An IFTS $(X, \tau)$ is IF-connected if and only if there exists no non-empty IFOS A and $B$ in $X$ such that $A=B^{C}$.

Proof. Necessity. Assume that $A$ and $B$ are two IFOSs in $X$ such that $A \neq(0,1) \neq B$ and $A=B^{C}$, since $B$ is an IFOS which implies that $B^{C}=A$ is an IFCS and $B \neq(0,1)$ implies that $B^{C} \neq(1,0)$, i.e., $A \neq(1,0)$. Hence there exists a proper IFS $A$ as $A \neq(0,1)$ and $A \neq(1,0)$, such that $A$ is both IFOS and IFCS. But this is a contradiction that $(X, \tau)$ is IF-connected.

Sufficiency. Let $(X, \tau)$ is an IFTS and A is both IFOS and IFCS in $X$ such that $(0,1) \neq A \neq$ $(1,0)$. Here $A=B^{C}$. In this case $B$ is an IFOS and $A \neq(1,0)$. This implies that, $B=A^{C} \neq(0,1)$, which is a contradiction. Hence, there exist no proper IFS in $X$ which is both IFO and IFC. So, $(X, \tau)$ is IF-connected.

Theorem 3.7. Let $(X, T)$ be a topological space and $(X, \tau)$ be its corresponding IFTS, where $\tau=\left\{\left(1_{A}, 1_{A^{C}}\right): A \in T\right\}$. Then $(X, T)$ is connected if and only if $(X, \tau)$ is IF-connected.

Proof. Suppose $(X, T)$ is disconnected, so there exist two non-empty subsets $A, B$ of $X$ such that $A \cup B=X, A \cap B=\emptyset$. Since $A, B \in T$ then $1_{A}=\left(1_{A}, 1_{A} C\right) \in \tau$ and $1_{B}=\left(1_{B}, 1_{B} C\right) \in \tau$.

$$
\begin{aligned}
& \text { Now, } 1_{A} \cup 1_{B}=\left(1_{A}, 1_{A} C\right) \cup\left(1_{B}, 1_{B} C\right) \\
& =\left(1_{A} \cup 1_{B}, 1_{A}^{C} \cap 1_{B} C\right) \\
& =\left(1_{A \cup B}, 1_{A} C_{\cap B} C\right) \\
& =\left(1_{A \cup B}, 1_{(A \cup B)^{C}}\right) \\
& =\left(1_{X}, 1_{\varnothing}\right) \\
& =(1,0) \text {. } \\
& \text { Again, } 1_{A} \cap 1_{B}=\left(1_{A}, 1_{A} c\right) \cap\left(1_{B}, 1_{B} C\right) \\
& =\left(1_{A} \cap 1_{B}, 1_{A} C \cup 1_{B} C\right) \\
& =\left(1_{A \cap B}, 1_{A} C_{\cup B} C\right) \\
& =\left(1_{A \cap B}, 1_{(A \cap B)^{C}}\right) \\
& =\left(1_{\varnothing}, 1_{X}\right) \\
& =(0,1) \text {. }
\end{aligned}
$$

So, $(X, \tau)$ is IF-disconnected. Hence $(X, T)$ is connected if $(X, \tau)$ is IF-connected.

Conversely, suppose $(X, \tau)$ is IF-disconnected. Since $1_{A}, 1_{B} \in \tau$ so $\left.m m, 0\right)$ and $1_{\mathrm{A}} \cap 1_{\mathrm{B}}=$ $(0,1)$, then we can write $1_{A} \cup 1_{B}=(1,0)$ 


$$
\begin{aligned}
& \Rightarrow\left(1_{A}, 1_{A^{C}}\right) \cup\left(1_{B}, 1_{B^{C}}\right)=(1,0) \\
& \Rightarrow\left(1_{A} \cup 1_{B}, 1_{A^{C}} \cap 1_{B^{C}}\right)=(1,0) \\
& \Rightarrow\left(1_{A \cup B}, 1_{A^{C} \cap B^{C}}\right)=(1,0)
\end{aligned}
$$

So, $1_{A \cup B}=1=1_{X} \Rightarrow 1^{-1}(0,1]=A \cup B=X$.

Again, $1_{A} \cap 1_{B}=(0,1)$.

$$
\begin{aligned}
& \Rightarrow\left(1_{A}, 1_{A^{C}}\right) \cap\left(1_{B}, 1_{B^{C}}\right)=(0,1) \\
& \Rightarrow\left(1_{A} \cap 1_{B}, 1_{A^{C}} \cup 1_{B^{C}}\right)=(0,1) \\
& \Rightarrow\left(1_{A \cap B}, 1_{A^{C} \cup B^{C}}\right)=(0,1) .
\end{aligned}
$$

So, $1_{A \cap B}=0=1_{\emptyset} \Rightarrow 1^{-1}(0,1]=A \cap B=\emptyset$. Hence $(X, T)$ is disconnected.

So, $(X, T)$ is connected if and only if $(X, \tau)$ is IF-connected.

Theorem 3.8. If $(X, \tau)$ and $(Y, \delta)$ are IF-connected space then $(X \times Y, \tau \times \delta)$ is also IFconnected.

Proof. Consider $(X \times Y, \tau \times \delta)$ is not IF-connected then $\exists A, B \in \tau \times \delta$ such that $A \cup B=(1,0)$ and $A \cap B=(0,1)$. Since $A, B \in \tau \times \delta$ then $A=C \times D$ and $B=E \times F$, where $C=\left(\mu_{C}, v_{C}\right)$, $E=\left(\mu_{E}, v_{E}\right) \in \tau$ and $D=\left(\mu_{D}, v_{D}\right), F=\left(\mu_{F}, v_{F}\right) \in \delta$. Now $C \times D=\left(\mu_{C} \times \mu_{D}, v_{C \times} \cdot v_{D}\right)$, where $\left(\mu_{C} \times \mu_{D}\right)(x, y)=\min \left(\mu_{C}(x), \mu_{D}(y)\right)$ and $\left(v_{C} \dot{x} v_{D}\right)(x, y)=\max \left(v_{C}(x), v_{D}(y)\right), \quad \forall(x, y) \in$ $\tau \times \delta$. Similarly, $E \times F=\left(\mu_{E} \times \mu_{F}, v_{E \times} \cdot v_{F}\right)$.

Now, $A \cup B=(1,0) \Rightarrow(C \times D) \cup(E \times F)=(1,0)$

$\Rightarrow\left(\mu_{C} \times \mu_{D}, v_{C \times} v_{D}\right) \cup\left(\mu_{E} \times \mu_{F}, v_{E \times} \cdot v_{F}\right)=(1,0)$

$\Rightarrow\left(\min \left(\mu_{C}(x), \mu_{D}(y)\right) \cup \min \left(\mu_{E}(x), \mu_{F}(y)\right), \max \left(v_{C}(x), v_{D}(y)\right) \cap \max \left(v_{E}(x), v_{F}(y)\right)\right)=$ $(1,0)$

i.e., $\min \left(\mu_{C}(x), \mu_{D}(y)\right) \cup \min \left(\mu_{E}(x), \mu_{F}(y)\right)=1$

$\Rightarrow$ Either, $\min \left(\mu_{C}(x), \mu_{D}(y)\right)=1$ or, $\min \left(\mu_{E}(x), \mu_{F}(y)\right)=1$

$\Rightarrow$ Either $\mu_{C}(x)=1, \mu_{D}(y)=1$ or, $\mu_{E}(x)=1, \mu_{F}(y)=1$

For, $\max \left(v_{C}(x), v_{D}(y)\right) \cap \max \left(v_{E}(x), v_{F}(y)\right)=0$

$\Rightarrow \max \left(v_{C}(x), v_{D}(y)\right)=0$ and $\max \left(v_{E}(x), v_{F}(y)\right)=0$

$\Rightarrow v_{C}(x)=0, v_{D}(y)=0, v_{E}(x)=0, v_{F}(y)=0$

Case I: Suppose $\mu_{C}(x)=1, \mu_{D}(y)=1$.

Then $C \cup E=\left(\mu_{C}, v_{C}\right) \cup\left(\mu_{E}, v_{E}\right)=\left(\mu_{C} \cup \mu_{E}, v_{C} \cap v_{E}\right)=(1,0)$ as $\mu_{C}(x)=1$.

Case II: Suppose $\mu_{E}(x)=1, \mu_{F}(y)=1$.

Then $D \cup F=\left(\mu_{D}, v_{D}\right) \cup\left(\mu_{F}, v_{F}\right)=\left(\mu_{D} \cup \mu_{F}, v_{D} \cap v_{F}\right)=(1,0)$ as $\mu_{F}(y)=1$.

Again, $A \cap B=(0,1) \Rightarrow(C \times D) \cap(E \times F)=(0,1)$

$\Rightarrow\left(\mu_{C} \times \mu_{D}, v_{C \times} v_{D}\right) \cap\left(\mu_{E} \times \mu_{F}, v_{E \times} v_{F}\right)=(1,0)$

$\Rightarrow\left(\min \left(\mu_{C}(x), \mu_{D}(y)\right) \cap \min \left(\mu_{E}(x), \mu_{F}(y)\right), \max \left(v_{C}(x), v_{D}(y)\right) \cup \max \left(v_{E}(x), v_{F}(y)\right)\right)=$ $(0,1)$,

i.e., $\min \left(\mu_{C}(x), \mu_{D}(y)\right) \cap \min \left(\mu_{E}(x), \mu_{F}(y)\right)=0$

$\Rightarrow \min \left(\mu_{C}(x), \mu_{D}(y)\right)=0$ and $\min \left(\mu_{E}(x), \mu_{F}(y)\right)=0$

$\Rightarrow$ Either $\mu_{C}(x)=0$, or $\mu_{D}(y)=0$ and either $\mu_{E}(x)=0$ or $\mu_{F}(y)=0$ 
Again, for, $\max \left(v_{C}(x), v_{D}(y)\right) \cup \max \left(v_{E}(x), v_{F}(y)\right)=1$

$\Rightarrow$ Either $\max \left(v_{C}(x), v_{D}(y)\right)=1$ or, $\max \left(v_{E}(x), v_{F}(y)\right)=1$

$\Rightarrow$ Either $v_{C}(x)=1$ or $v_{D}(y)=1$, or, either $v_{E}(x)=1$ or $v_{F}(y)=1$

Case III: Suppose $\mu_{C}(x)=0$, or $\mu_{D}(y)=0$ and $v_{C}(x)=1$.

Then $C \cap E=\left(\mu_{C}, v_{C}\right) \cap\left(\mu_{E}, v_{E}\right)=\left(\mu_{C} \cap \mu_{E}, v_{C} \cup v_{E}\right)=(0,1)$.

Case IV: Suppose $\mu_{E}(x)=0$ or $\mu_{F}(y)=0$ and $v_{F}(y)=1$.

Then $D \cap F=\left(\mu_{D}, v_{D}\right) \cap\left(\mu_{F}, v_{F}\right)=\left(\mu_{D} \cap \mu_{F}, v_{D} \cup v_{F}\right)=(0,1)$.

So, $(X, \tau)$ and $(Y, \delta)$ are not connected, hence if $(X, \tau)$ and $(Y, \delta)$ are IF-connected then $(X \times Y, \tau \times \delta)$ is IF-connected.

Definition 3.6. An IFTS $(X, \tau)$ is said to be totally IF-connected if for each pair of IFP $p_{\alpha, \beta}, q_{\rho, \theta} \in X$, there exists a disconnection $G \cup H$ of $X$ with $p_{\alpha, \beta} \in G$ and $q_{\rho, \theta} \in H$.

Theorem 3.9. The continuous image of a totally IF-disconnected space is totally IFdisconnected.

Proof. Let $f:(X, \tau) \rightarrow(Y, \delta)$ be a continuous function from an $\operatorname{IFTS}(X, \tau)$ to $(Y, \delta)$. Consider $x_{\alpha, \beta}, y_{r, s}$ be two IFP in $Y=f(X)$. Since $f$ is continuous $f^{-1}\left(x_{\alpha, \beta}\right)$ and $f^{-1}\left(y_{r, s}\right)$ are IFP in $X$. If $(X, \tau)$ is totally IF-disconnected then there exists a disconnection $G \cup H$ of $X$ where $f^{-1}\left(x_{\alpha, \beta}\right) \in G=\left(\mu_{G}, v_{G}\right)$ and $f^{-1}\left(y_{r, s}\right) \in H=\left(\mu_{H}, v_{H}\right)$. Since $f^{-1}\left(x_{\alpha, \beta}\right) \in G \Rightarrow x_{\alpha, \beta} \in f(G)$ and $f^{-1}\left(y_{r, s}\right) \in H \Rightarrow y_{r, s} \in f(H)$. Again $G \cup H$ is a disconnection of $X$ such that $G \cup H=$ $(1,0)$ and $\cap H=(0,1)$.

Here, $G \cup H=(1,0) \Rightarrow\left(\mu_{G}, v_{G}\right) \cup\left(\mu_{H}, v_{H}\right)=(1,0)$

$\Rightarrow\left(\mu_{G} \cup \mu_{H}, v_{G} \cap v_{H}\right)=(1,0)$

And $G \cap H=(0,1) \Rightarrow\left(\mu_{G}, v_{G}\right) \cap\left(\mu_{H}, v_{H}\right)=(1,0)$

$\Rightarrow\left(\mu_{G} \cap \mu_{H}, v_{G} \cup v_{H}\right)=(1,0)$.

So, $f(G)=\left(f\left(\mu_{G}\right), f\left(v_{G}\right)\right)$ and $f(H)=\left(f\left(\mu_{H}\right), f\left(v_{H}\right)\right)$ gives

$f(G) \cup f(H)=\left(f\left(\mu_{G}\right), f\left(v_{G}\right)\right) \cup\left(f\left(\mu_{H}\right), f\left(v_{H}\right)\right)$

$=\left(f\left(\mu_{G}\right) \cup f\left(\mu_{H}\right), f\left(v_{G}\right) \cap f\left(v_{H}\right)\right)$

$=\left(\left(\mu_{G} \cup \mu_{H}\right)\left(f^{-1}(x)\right),\left(v_{G} \cap v_{H}\right)\left(f^{-1}(x)\right)\right)$

$=(1,0)$.

And $f(G) \cap f(H)=\left(f\left(\mu_{G}\right), f\left(v_{G}\right)\right) \cap\left(f\left(\mu_{H}\right), f\left(v_{H}\right)\right)$

$=\left(f\left(\mu_{G}\right) \cap f\left(\mu_{H}\right), f\left(v_{G}\right) \cup f\left(v_{H}\right)\right)$

$=\left(\left(\mu_{G} \cap \mu_{H}\right)\left(f^{-1}(x)\right),\left(v_{G} \cup v_{H}\right)\left(f^{-1}(x)\right)\right)$

$=(0,1)$.

So, $Y=f(X)$ is totally IF-disconnected.

Definition 3.7. An IFTS $(X, \tau)$ is $T_{1}$-space if $\forall$ IF-singleton $x_{\alpha, \beta}, y_{m, n} \in X$ with $x_{\alpha, \beta} \neq y_{m, n}$, then $\exists A=\left(\mu_{A}, v_{A}\right), B=\left(\mu_{B}, v_{B}\right) \in \tau$ such that $x_{\alpha, \beta} \in A, y_{m, n} \notin A$ and $x_{\alpha, \beta} \notin B, y_{m, n} \in B$.

Theorem 3.10. Every $I F-T_{1}$ space is totally IF-disconnected space.

Proof. Let $(X, \tau)$ be an IFTS and also IF- $T_{1}$ space. If $x_{\alpha, \beta}, y_{m, n} \in X$ with $x_{\alpha, \beta} \neq y_{m, n}$ then $\exists A=\left(\mu_{A}, v_{A}\right), B=\left(\mu_{B}, v_{B}\right) \in \tau$ such that $x_{\alpha, \beta} \in A, y_{m, n} \notin A$ and $x_{\alpha, \beta} \notin B, y_{m, n} \in B$.

Now

$$
\begin{aligned}
& x_{\alpha, \beta} \in A=\left(\mu_{A}, v_{A}\right) \Rightarrow \mu_{A}(x) \geq \alpha, v_{A}(x) \leq \beta, \\
& x_{\alpha, \beta} \notin B=\left(\mu_{B}, v_{B}\right) \Rightarrow \mu_{B}(x)<\alpha, v_{B}(x)>\beta,
\end{aligned}
$$




$$
\begin{aligned}
& y_{m, n} \notin A=\left(\mu_{A}, v_{A}\right) \Rightarrow \mu_{A}(y)<m, v_{A}(y)>n, \\
& y_{m, n} \in B=\left(\mu_{B}, v_{B}\right) \Rightarrow \mu_{B}(y) \geq m, v_{B}(y) \leq n .
\end{aligned}
$$

So, $(A \cup B)(x)=\left(\mu_{A} \cup \mu_{B}, v_{A} \cap v_{B}\right)>(\alpha, \beta),(A \cap B)(x)=\left(\mu_{A} \cap \mu_{B}, v_{A} \cup v_{B}\right)<(\alpha, \beta)$ and $(A \cup B)(y)=\left(\mu_{A} \cup \mu_{B}, v_{A} \cap v_{B}\right)>(m, n),(A \cap B)(y)=\left(\mu_{A} \cap \mu_{B}, v_{A} \cup v_{B}\right)<(m, n)$. Hence $A \cup B$ is a disconnection of $X$, so $(X, \tau)$ is totally IF-disconnected.

\section{Conclusion}

The results presented in this paper indicate that many of the basic concepts in general topology can readily to extend to intuitionistic fuzzy topological spaces. Although the theory of intuitionistic fuzzy set is still in embryonic stage, it shows promise of having wide applications

\section{Acknowledgements}

The authors wish to thank the reviewer for his suggestions and corrections which helped to improve this paper.

\section{References}

[1] Ahmed, E., Hossain, M. S., \& Ali, D. M. (2014). On Intuitionistic Fuzzy $T_{0}$ Spaces. Journal of Bangladesh Academy of Sciences, 38(2) 197-207.

[2] Ahmed, E., Hossain, M. S., \& Ali, D. M. (2015). On Intuitionistic Fuzzy $R_{0}$ Spaces. Annals of Pure and Applied Mathematics, 10(1), 7-14.

[3] Ahmed, E., Hossain, M. S., \& Ali, D. M. (2015). On Intuitionistic Fuzzy $R_{1}$ Spaces. Journal of Mathematics and Computer Science, 5(5), 681-693.

[4] Ahmed, E., Hossain, M. S., \& Ali, D. M. (2014). On Intuitionistic Fuzzy $T_{1}$ Spaces. Journal of Physical Sciences, 19, 59-66.

[5] Ahmed, E., Hossain, M. S., \& Ali, D. M. (2014). On Intuitionistic Fuzzy $T_{2}$ Spaces. IOSR Journal of Mathematics, 10(6), 26-30.

[6] Ahmad, M. K., \& Salahuddin, M. (2013). Fuzzy Generalized Variational Like Inequality Problems in Topological Vector Spaces. Journal of Fuzzy Set Valued Analysis, 2013(1), $1-5$.

[7] Ali, A. M., Senthil, S., \& Chendralekha, T. (2016). Intuitionistic Fuzzy Sequences in Metric Space. International Journal of Mathematics and its Applications, 4(1-B), 155-159.

[8] Ali, A. M., \& Kanna, G. R. (2017). Intuitionistic Fuzzy Cone Metric Spaces and Fixed Point Theorems. International Journal of Mathematics and its Applications, 5(1-A), 25-36.

[9] Atanassov, K.T. (1986). Intuitionistic fuzzy sets. Fuzzy Sets and Systems, 20(1), 87-96. 
[10] Barile, M. $T_{0}$ space. Wolfram Mathworld. Available online at: https://mathworld. wolfram.com/T0-Space.html

[11] Bayhan, S., \& Coker, D. (1996). On fuzzy separation axioms in intuitionistic fuzzy topological space. BUSEFAL, 67, 77-87.

[12] Bayhan, S., Coker, D. (2005). Pairwise separation axioms in intuitionistic topological Spaces. Hacettepe Journal of Mathematics and Statistics, 34 S, 101-114.

[13] Chang, C. L. (1968). Fuzzy Topological Space. Journal of Mathematical Analysis and Application, 24, 182-190.

[14] Coker, D. (1996). A note on intuitionistic sets and intuitionistic points. Turkish Journal of Mathematics, 20(3), 343-351.

[15] Coker, D. (1997). An introduction to intuitionistic fuzzy topological spaces. Fuzzy Sets and Systems, 88(1), 81-89.

[16] Coker, D., \& Bayhan, S. (2001). On Separation Axioms in Intuitionistic Topological Space. International Journal of Mathematics and Mathematical Sciences, 27(10), 621-630.

[17] Coker, D., \& Bayhan, S. (2003). On $T_{1}$ and $T_{2}$ Separation Axioms in Intuitionistic fuzzy Topological Space. Journal of Fuzzy Mathematics, 11(3), 581-592.

[18] Das, S. (2013). Intuitionistic Fuzzy Topological Spaces (MS Thesis Paper). Dept. of Math, National Inst. of Tech., India.

[19] Fang, J., \& Guo, Y. (2012). Quasi-coincident neighbourhood structure of relative I-fuzzy topology and its applications. Fuzzy Sets and Systems, 190, 105-117.

[20] Hassan, Q. E. (2007). On some kinds of fuzzy connected spaces. Applications of Mathematics, 52(4), 353-361.

[21] Immaculate, H. J., \& Arockiarani, I. (2015). A new class of connected spaces in intuitionistic topological spaces. International Journal of Applied Research, 1(9), 720-726.

[22] Islam, R., Hossain, M. S., \& Hoque, M. F. (2020). A study on $L$-fuzzy $T_{1}$ Spaces. Notes on Intuitionistic Fuzzy Sets, 26(3), 33-42.

[23] Islam, M. S., Hossain, M. S., \& Asaduzzaman, M. (2017). Level Seperation on Intuitionistic Fuzzy $T_{0}$ spaces. International Journal of Fuzzy Mathematical Archive, 13(2), 123-133.

[24] Islam, M. S., Hossain, M. S., \& Asaduzzaman, M. (2018). Level separation on Intuitionistic fuzzy $T_{2}$ spaces. Journal of Mathematical and Computational Science, 8(3), 353-372.

[25] Lee, S. J., \& Lee, E. P. (2000). The Category of Intuitionistic Fuzzy Topological Space. Bulletin of the Korean Mathematical Society, 37(1), 63-76.

[26] Lee, S. J., \& Lee, E. P. (2004). Intuitionistic Fuzzy Proximity Spaces. International Journal of Mathematics and Mathematical Sciences, 49, 2617-2628. 
[27] Mahbub, M. A., Hossain, M. S., \& Hossain, M. A. (2018). Some Properties of Compactness in Intuitionistic Fuzzy Topological Spaces. International Journal of Fuzzy Mathematical Archive, 16(1), 39-48.

[28] Minana, J. J., \& Sostak, A. (2016). Fuzzifying topology induced by a strong fuzzy metric. Fuzzy Sets and Systems, 300, 24-39.

[29] Ozcag, S., \& Coker, D. (1998). On connectedness in intuitionistic fuzzy special topological spaces. International Journal of Mathematics and Mathematical Sciences, 21(1), 33-40.

[30] Ramadan, A. A., Abbas, S. E., \& Abd El-Latif, A. A. (2005). Compactness in Intuitionistic Fuzzy Topological Spaces. International Journal of Mathematics and Mathematical Sciences, 2005(1), 19-32.

[31] Sethupathy, K. S. R., \& Lakshmivarahan, S. (1977). Connectedness in Fuzzy Topology. Kybernetika, 13(3), 190-193.

[32] Singh, A. K., \& Srivastava, R. (2012). Separation Axioms in Intuitionistic Fuzzy Topological Spaces. Advances in Fuzzy Systems, 2012, Article ID 604396.

[33] Srivastava, S., Lal, S. N., \& Srivastava, A.K. (1988). On fuzzy $T_{0}$ and $R_{0}$ topological spaces. Journal of Mathematical Analysis and Application, 136(1), 66-73.

[34] Srivastava, R., \& Singh, A. K. (2011). Connectedness in intuitionistic fuzzy topological spaces. Comptes rendus de 1'Academie bulgare des Sciences, 64(9), 1241-1250.

[35] Talukder, M. A. M., \& Ali, D. M. (2013). Some Features of Fuzzy $\alpha$-Compactness. International Journal of Fuzzy Mathematical Archive, 2, 85-91.

[36] Tan, N. X. (1985). Quasi variational inequalities in topological linear locally convex Hausdorff spaces. Mathematische Nachrichten, 122, 231-245.

[37] Tamilmani, J. (2015). Presemi Weakly Closed Set in Intuitionistic Fuzzy Topological Spaces. International Journal of Fuzzy Mathematical Archive, 6(1), 27-34.

[38] Tiwari, S. P., Srivastava, A. K. (2013). Fuzzy rough sets, fuzzy preorders and fuzzy topologies. Fuzzy Sets and Systems, 210, 63-68.

[39] Ying-Ming, L., \& Mao-Kang, L. (1997). Fuzzy Topology. World Scientific Publishing Co. Pte. Ltd.

[40] Zadeh, L. A. (1965). Fuzzy sets. Information and Control, 8(3), 338-353. 